\title{
Efficacy of inverted internal limiting membrane flap for large idiopathic macular holes
}

\author{
Haroon Tayyab ${ }^{1}$, Asad Aslam Khan², Sana Jahangir ${ }^{3}$
}

\begin{abstract}
Objective: To evaluate the effectiveness of inverted internal limiting membrane flap in large idiopathic macular holes.

Methods: Twelve patients diagnosed with idiopathic macular holes larger than 400um underwent 23 gauge Pars Plana Vitrectomy (PPV) with inverted internal limiting flap and gas tamponade at Al-Ehsan Eye Welfare Eye Hospital, Lahore from February 2017 to February 2018. All cases were diagnosed on Spectral Domain Optical Coherence Tomography (SD-OCT) and were followed up for 6 months. At every follow-up, best corrected visual acuity and SD-OCT was done.

Results: We achieved macular hole closure in 91.6\% (11/12) patients with idiopathic macular holes larger than 400um. Five out of 12 patients underwent combined phacoemulsification and PPV. One patient has flat hole closure which was considered failure. One patient was excluded from the study due to per-operative flap loss. This patient was not included in final data analysis of 12 patients. There was statistically significant gain in best corrected visual acuity after successful macular hole closure. We did not report any untoward events during or in the post-operative period.
\end{abstract}

Conclusion: Inverted internal limiting flap is an effective method for repairing large macular holes.

KEYWORDS: Idiopathic macular hole, Internal limiting membrane, Pars plana vitrectomy.

How to cite this:

doi: https://doi.org/10.12669/pjms.35.2.689

Tayyab H, Khan AA, Jahangir S. Efficacy of inverted internal limiting membrane flap for large idiopathic macular holes. Pak J Med Sci. 2019;35(2):315-319. doi: https://doi.org/10.12669/pjms.35.2.689

This is an Open Access article distributed under the terms of the Creative Commons Attribution License (http://creativecommons.org/licenses/by/3.0), which permits unrestricted use, distribution, and reproduction in any medium, provided the original work is properly cited.

\section{INTRODUCTION}

1. Dr. Haroon Tayyab, FCPS (Ophth), FCPS (VRO),

2. Prof. Asad Aslam Khan, MS, FCPS, PhD,

3. Dr. Sana Jahangir, FCPS,

Department of Ophthalmology,

Sharif Medical \& Dental College, Lahore, Pakistan.

1,2: Department of Ophthalmology,

College of Ophthalmology \& Allied Vision Sciences,

King Edward Medical University,

Lahore, Pakistan.

Correspondence:

Dr. Haroon Tayyab, FCPS (Ophth), FCPS (VRO), Assistant Professor of Vitreoretinal Surgery, Eye Unit 3, COAVS,

King Edward Medical University,

Mayo Hospital, Lahore, Pakistan.

Email: haroontayyab79@gmail.com

* Received for Publication:

September 11, 2018

* $1^{\text {st }}$ Revision Received:

* $2^{\text {nd }}$ Revision Received:

* Final Revision Accepted:
September 26, 2018

February 15, 2019

February 18, 2019
Idiopathic Macular Hole (IMH) is one of the most widely studied vitreoretinal interface pathology. ${ }^{1}$ Its reported incidence is 7.8 / 100,000 per annum with women being more commonly effected as compared to males. ${ }^{1,2}$ Before 1991, macular hole was considered an untreatable condition. It was Kelly and Wendel who for the first time reported Pars Plana Vitrectomy (PPV) and fluid gas exchange as a procedure of choice for IMH. They reported anatomical success in $58 \%$ of patient and functional success in $42 \%$ of patients in their pilot study. ${ }^{3}$

In further studies, they improved upon their results as their understanding of pathogenesis behind macular hole improved. ${ }^{4}$ Afterwards, various modifications were introduced in IMH surgery and the most notable was Internal Limiting Membrane (ILM) peeling. With ILM peeling, structural success of more than $90 \%$ was 
reported in various studies., ${ }^{5,6}$ Removal of ILM around the macular holes allows the retina to restore back to its inherent elastic state by removal of tangential centripetal traction around the hole edge. This technique has very good anatomic and functional results when the IMH minimum diameter is small.

When dealing with larger macular holes, the structural and functional success declines and also sometimes requiring reoperations. ${ }^{7}$ The most desirable macular hole closure is U-shaped closure with best visual results. In 20 to $40 \%$ of cases, surgeons achieve a W-shaped closure with exposed Retinal Pigment Epithelium (RPE). Although it is considered a structural success but visual gains are limited. $^{8}$

In 2010, Michalewska introduced a novel technique in inverted ILM flap for closure of macular holes larger than $400 \mathrm{um}$. He reported excellent structural and functional results later on. The rationale for his technique was to promote glial cell proliferation; thus plugging the macular hole and having some improvement in functional results too. ${ }^{9}$ As the technique gained popularity, more ambitious studies showed that inverted ILM peel technique was also successful in IMH larger than 1000um. ${ }^{10,11}$ These results showed that those macular holes that may have been left untreated due to their large size have a potential to benefit from this technique.

Other significant advances have been the introduction of different staining agents for staining ILM. The first one was indocyanine green dye followed by trypan blue and brilliant blue G dye. These vital dyes have made staining and peeling of ILM very easy and relatively safe in modern vitreoretinal surgery. ${ }^{12,13}$

Since there has been no local study to evaluate the results of inverted ILM peel in large macular holes, we attempted to assess the outcome in our local settings and share our experience regarding this technique.

\section{METHODS}

This was a prospective interventional study conducted in vitreo-retina unit of Al- Ehsan Welfare Eye Hospital, Lahore, Pakistan (a tertiary care Ophthalmic facility) from February 2017 to February 2018. A total of 12 patients were enrolled. All patients were informed about the nature of disease and type of intervention being planned for their eyes. A written informed consent was obtained from all patients. Permission for this study was sought from Ethics Committee of the hospital. Patients with IMH larger than 400um were included in this study. Patients who had any other concurrent ocular pathology like retinal vascular diseases (diabetic retinopathy, retinal vein occlusion etc), age related macular degeneration, glaucoma, history of previous retinal surgery, history of trauma, uveitis, solar retinopathy and other vitreo-retinal interface problems were excluded from this study. Patients with high myopia (> 6 Diopter Sphere) or IMH with retinal detachment were also not included in this study. All phakic patients were simultaneously operated for cataract with foldable intraocular lens implant.

Best corrected visual acuity (BCVA) was recorded using Early Treatment Diabetic Retinopathy Study (ETDRS) type LogMAR chart. We evaluated IMH using spectral domain optical coherence tomography (Topcon 3D OCT-1 Maestro. Topcon Europe Medical BV Essebaan 11, 2908 LJ, Capelle aan den IJssel, The Netherlands). Macular holes with minimum diameter of $>400$ um were included in this study. Intraocular pressure was measured using standard Goldmann applanation tonometry (AT 900 - Applanation tonometer, Haag Streit AG, Germany). This examination was carried out before the surgery and 45 days after surgery to allow sufficient resolution of intraocular gas for convenient SD-OCT evaluation.

Surgical Technique: All cases were performed using 23 gauge three port transconjunctival PPV by a single experienced vitreoretinal surgeon. Faros vitrectomy system (Oertli Instrumente AG, Switzerland) was used in all cases. After entry in vitreous cavity through $23 \mathrm{G}$ trocar system implanted at $3.5 \mathrm{~mm}$ from limbus, core vitrectomy was performed. We used $0.1 \mathrm{ml}$ triamcinolone acetonide $(40 \mathrm{mg} / \mathrm{ml})$ to stain posterior cortical vitreous and for induction of Posterior Vitreous Detachment (PVD). After complete vitrectomy, fluid air exchange was performed. Twin dye (TWIN 018 HD $0.18 \%$ trypan blue $+0.03 \%$ blulife, AL. CHI.MI.A. SRL - Viale Austria) was instilled on macula under air and left in place for 30 seconds for adequate staining. After washing out the dye and filling vitreous cavity with basic salt solution, ILM peel under high magnification was attempted. We used Oculus BIOM 2 with Oculus SDI Inverter 2 (OCULUS Surgical, Inc. Port St. Lucie, USA) as preferred viewing system in all cases. Grieshaber 23G disposable ILM peeling forceps were employed using pinch and peel technique for grasping and peeling ILM. ILM peel was started superiorly with 


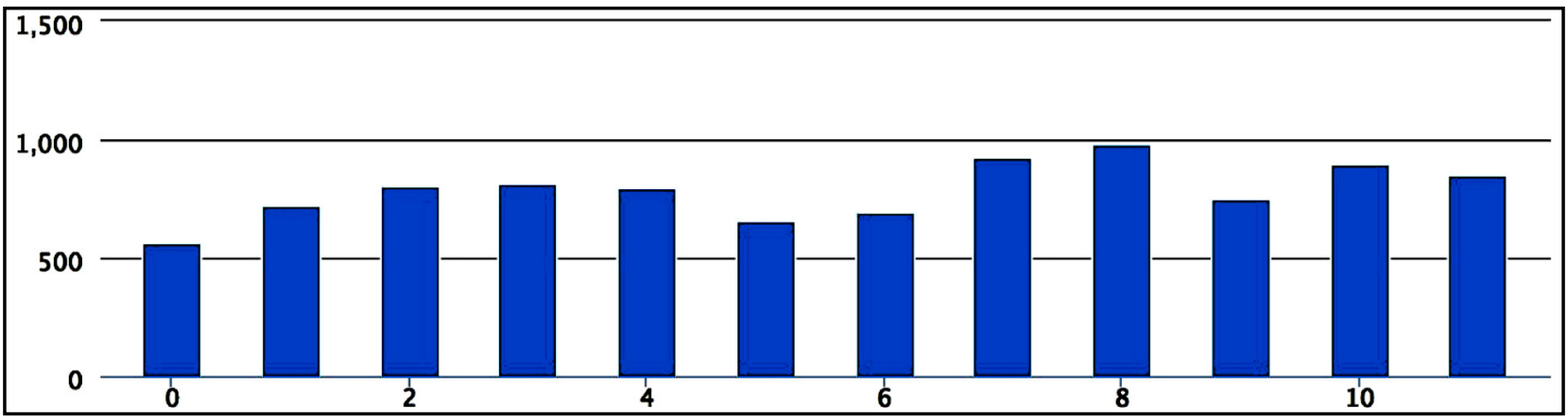

Fig.1: Shows minimum macular hole diameter in 12 study cases. $X$ axis shows macular hole diameter in um.

a diameter of 2 discs around macular hole in such a way that only a thing rim remained attached to macular hole margins with a pedicle.

During this maneuver, perfusion was set at a low level. The ILM flapedges were trimmed with vitreous cutter using high cut rate (4000 cuts / min) and very low vacuum (50). Under low infusion pressures, ILM flap that remained attached to macular hole margins was massaged over the macular hole using silicon tip backflush needle in such a way that the ILM side facing the vitreous cavity was now facing RPE and covering entire area of exposed RPE. No extra manipulation was done here after. After positioning the flap in inverted manner over the macular hole, standard fluid air gas (GOT Multi C3F8 - pure octafluoropropane gas. AL.CHI.MI.A. SRL - - Viale Austria) exchange was performed with $14 \% \mathrm{C} 3 \mathrm{~F} 8$ filling vitreous cavity at the conclusion of surgery. Trocars were withdrawn and any visible gas leak was addressed with 70 Vicryl suture. Face down post-operative positioning was instructed for seven days. A follow up schedule of 1st week, 1st month, $2^{\text {nd }}$ month and $6^{\text {th }}$ month (total study time of one year from February 2017 to February 2018) was followed for every patient. Macular holes were considered closed if they were close at 6 months after surgery. Flat open closure type configuration was considered as surgical failure.

Data analysis: For data analysis, we used SPSS software (version16.0; SPSS Inc., Chicago, Illinois, USA) for data analysis. Numerical data was represented as mean +/- standard deviation. Wilcoxon signed ranked test was used to assess statistical significance of pre and post-operative BCVA. $\quad \mathrm{P}<0.05$ was considered statistically significant.

\section{RESULTS}

A total of 12 eyes of 12 patients were included in this study. Eight (66.6\%) were females and 4 (33.3\%) were males. Mean age of patients in this study was $63.41 \pm 5.93$ years. Five out of 12 patients underwent simultaneous cataract extraction with foldable IOL implant. Using SD-OCT, the mean minimum width of IMH was $788 \pm 120.73$ um with range of 561 - 983 um. Individual values are shown in Fig.1. The mean duration of macular hole was $7.16 \pm 2.29$ months. The mean BCVA before surgery was 1.236 \pm 0.265 Log MAR units. Mean BCVA 2 months and 6 months after surgery was $0.989 \pm 0.298 \log M A R$ units and $0.918 \pm 0.336$ The difference between pre and post-operative BCVA at 2 months and 6 months was statistically significant ( $p$-value $<0.05$ ).

We had structural success in 11 out of 12 (91.66\%) cases. One IMH had Type-2 macular hole closure with flat macular hole edges but no retinal or glial tissue growth with exposed RPE (Fig.2). One case had loss of ILM flap during surgery and was not included in final analysis because only conventional ILM peel was accomplished in this case. We do not report any untoward events or side effects during or in the post-operative recovery period. Few cases with successful macular hole closure with inverted ILM flap are shown in Fig. 3 and 4 with pre operative and post operative OCTs stacked on top and bottom of figures respectively. Follow up period for post op OCTs was two months.

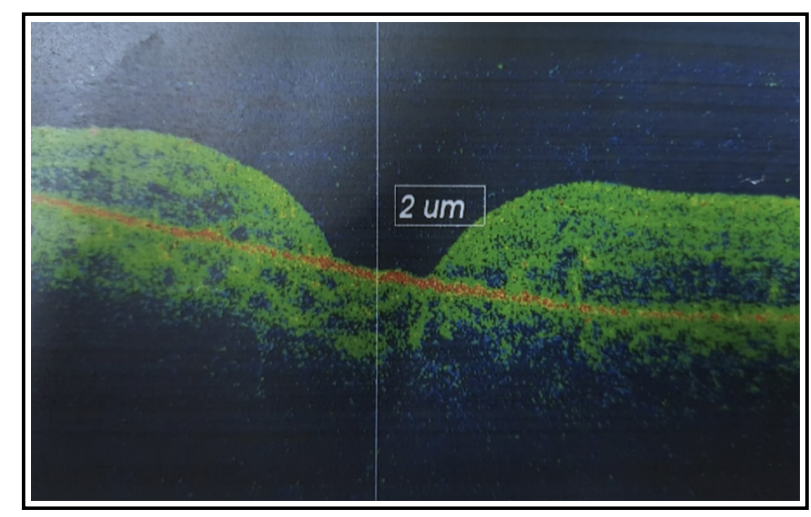

Fig.2: Type 2 macular hole closure. 


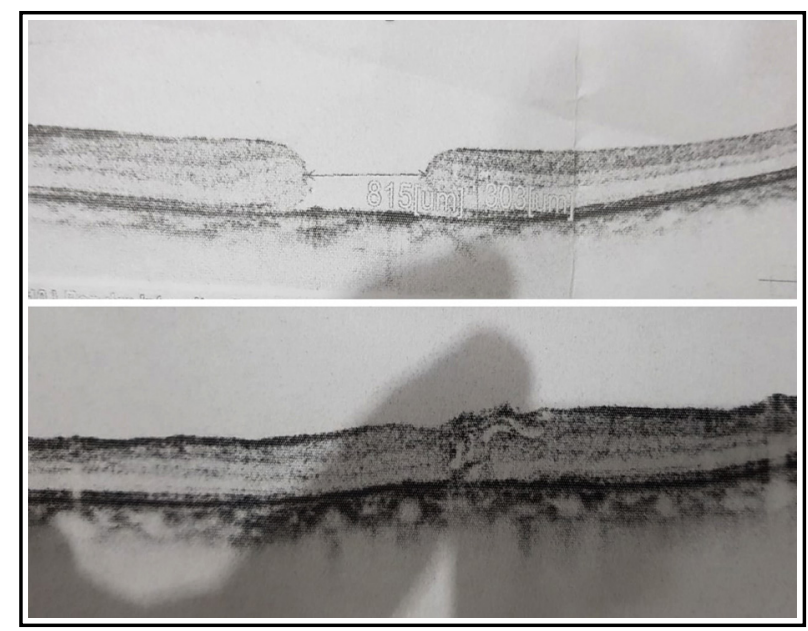

Fig.3: Shows pre-opertaive and post-operative OCTs of macular hole closure with inverted ILM flap.

\section{DISCUSSION}

It has been known through literature and experience that larger macular holes have relatively lower rate of successful closure and visual improvement and higher rates of reopening. ${ }^{14-16}$ It was Michalewska in 2010 who reported a novel approach of inverted ILM flap for closing macular holes larger than $400 \mathrm{um}$. He also reported significant visual improvement in his cases. ${ }^{9}$ Since then; there have been various reports on inverted ILM flap with or without some modifications. These modifications include pedicle ILM flap, autologous and pedicle ILM flap and double ILM flap. ${ }^{17-19}$ Surgeons have also successfully attempted inverted ILM flaps in cases of myopic macular holes and macular hole associated rhegmatogenous retinal detachment. ${ }^{16,20}$ In all these studies, researchers have taken a hole larger than 400um as large macular hole. Our inclusion criteria have also been the same. All patients included in this study had macular hole larger than 400um; although the IMH with minimum most diameter in this study was 561um. In a recent major review by Yamashita and Japan Clinical Retina Research Team (J-CREST), they reported $100 \%$ success rate for macular hole closure with inverted ILM flap as compared to conventional ILM peel. They reported 86\% (38/43) closure rate for holes $>550$ um with conventional ILM peel as compared to $100 \%(41 / 41)$ closure rate with inverted ILM flap. Their BCVA results are also comparable to our study. ${ }^{16}$ In another subgroup (macular holes $>700 \mathrm{um}$ ), the same team reported $69 \%(9 / 13)$ closure rate as compared to $100 \%(12 / 12)$ in conventional vs. inverted ILM flap respectively.

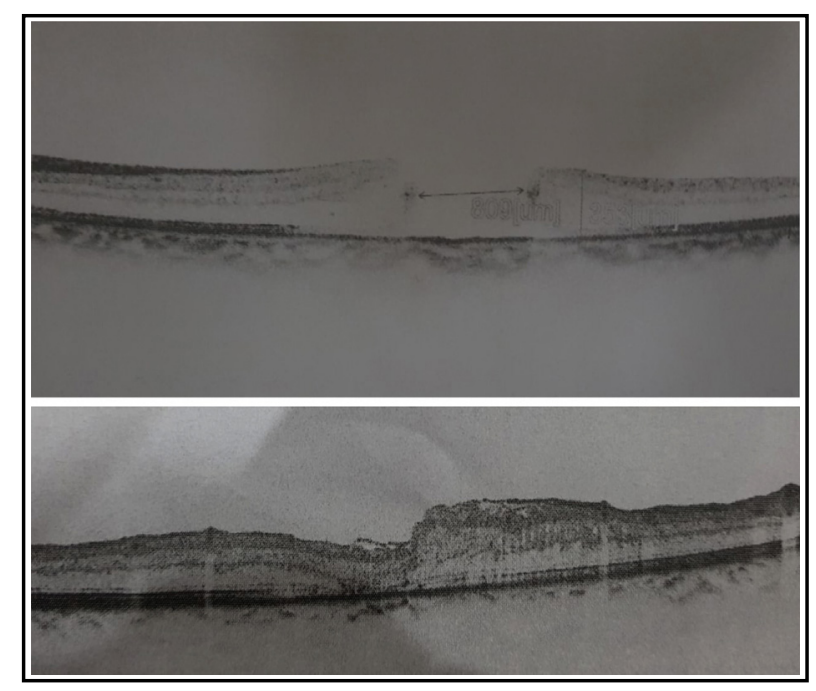

Fig.4: Shows pre-opertaive and post-operative OCTs of macular hole closure with inverted ILM flap.

In another large series published by Rizzo recently, he showed 96\% success in inverted ILM flap group versus $79 \%$ success in conventional group for IMH larger than 400um. They also reported similar success rate in BCVA as well. ${ }^{21}$ They also reported statistically significant results in favour of inverted ILM flap technique when considering eye with axial length $>26 \mathrm{~mm}(88 \%$ versus $39 \%$ ).

Similar results were reported in smaller series when Khodani reported 100\% (5/5) results with inverted ILM flap in macular holes with mean base diameter of $1420+/-84.8$ um. ${ }^{11}$ Mahalingham also reported $100 \%(5 / 5)$ results with inverted ILM flap technique with mean hole diameter of $811.4 \mathrm{um}{ }^{10}$

It was postulated by Michalewska that ILM flap provides mesh for proliferation of glial cells that eventually close the macular hole. This has been observed in many studies conducted in inverted macular flaps. It was further proposed after wards that the inverted flap not only provides a scaffold for glial cells to proliferate but also some stimulates the movement of photoreceptors towards the centre of fovea; thus providing neurosensory retina in the area of exposed RPE. ${ }^{9}$ In few of the cases it was shown in our study that inverted ILM flap actually stimulated the centripetal movement of photoreceptors to close the macular hole. (Fig.3 \& 4).

In our study, we had structural success in $11 / 12$ $(91.66 \%)$ cases with mean smallest diameter of macular hole being $788 \mathrm{um}$. We also report statically significant BCVA improvement. The strength of our study was its prospective design and a constant variant of ILM flap technique being performed by 
single surgeon with adequate follow-up period. The deficiencies include its small sample size with lack of control arm.

\section{CONCLUSION}

After extensive literature review and observation of our own results, we conclude that vitreoretinal surgeon should consider inverted ILM flap for large macular holes (>400um). Although, this technique is a brief learning curve but it can yield excellent anatomic outcome for macular holes previously considered as high risk for failure after undergoing conventional ILM peel. We recommend studies with multiple centres and more patients to evaluate the efficacy of this technique in our local setups. We also need to conduct study with control group undergoing conventional ILM peel for large macular holes to have more meaningful statistical results.

Grant Support \& Financial Disclosures: None.

\section{REFERENCES}

1. Chakrabarti $\mathrm{M}$, Benjamin $\mathrm{P}$, Chakrabarti $\mathrm{K}$, Chakrabarti A. Closing Macular Holes with "Macular Plug" without Gas Tamponade and Postoperative Posturing. Retina. 2017;37(3):451-459. doi: 10.1097/IAE.0000000000001206.

2. McCannel CA, Ensminger JL, Diehl NN, Hodge DN. Ophthalmology. 2009;116(7):1366-1369. doi: 10.1016/j. ophtha.2009.01.052.

3. Kelly NE, Wendel RT. Vitreous surgery for idiopathic macular holes. Results of a pilot study. Arch Ophthalmol. 1991;109(5):654-659.

4. Wendel RT, Patel AC, Kelly NE, Salzano TC, Wells JW, Novack GD. Vitreous surgery for macular holes. Ophthalmology. 1993;100(11):1671-1676.

5. Park DW, Sipperley JO, Sneed SR, Dugel PU, Jacobsen J. Macular hole surgery with internal-limiting membrane peeling and intravitreous air. Ophthalmology. 1999;106(7):1392-1397; discussion 1397-1398. doi: 10.1016/ S0161-6420(99)00730-7.

6. Chatziralli IP, Theodossiadis PG, Steel DHW. Internal Limiting Membrane Peeling in Macular Hole Surgery; Why, When, And How? Retina. 2018;38(5):870-882. doi: 10.1097/IAE.0000000000001959.

7. Michalewska Z, Michalewski J, Cisiecki S, Adelman $\mathrm{R}$, Nawrocki J. Correlation between foveal structure and visual outcome following macular hole surgery: a spectral optical coherence tomography study. Graefes Arch Clin Exp Ophthalmol. 2008;246(6):823-830. doi: 10.1007/s00417-007-0764-5.

8. Kang SW, Ahn K, Ham DI. Types of macular hole closure and their clinical implications. Br J Ophthalmol. 2003;87(8):1015-1019.

9. Michalewska Z, Michalewski J, Adelman RA, Nawrocki J. Inverted internal limiting membrane flap technique for large macular holes. Ophthalmology. 2010;117(10):20182025. doi: 10.1016/j.ophtha.2010.02.011.

10. Mahalingam P, Sambhav K. Surgical outcomes of inverted internal limiting membrane flap technique for large macular hole. Indian J Ophthalmol. 2013;61(10):601-603. doi: 10.4103/0301-4738.121090.
11. Khodani M, Bansal $P$, Narayanan $R$, Chhablani J. Inverted internal limiting membrane flap technique for very large macular hole. Int J Ophthalmol. 2016;9(8):1230-1232. doi: 10.18240/ijo.2016.08.22.

12. Beutel J, Dahmen G, Ziegler A, Hoerauf H. Internal limiting membrane peeling with indocyanine green or trypan blue in macular hole surgery: a randomized trial. Arch Ophthalmol. 2007;125(3):326-332.

13. Henrich PB, Haritoglou C, Meyer P, Ferreira PR, Schotzau A, Katamay R, et al. Anatomical and functional outcome in brilliant blue $G$ assisted chromovitrectomy. Acta Ophthalmol. 2010;88(5):588-593. doi: 10.1111/j.17553768.2008.01477.x.

14. Bae K, Kang SW, Kim JH, Kim SJ, Kim JM, Yoon JM. Extent of Internal Limiting Membrane Peeling and its Impact on Macular Hole Surgery Outcomes: A Randomized Trial. Am J Ophthalmol. 2016;169:179-188. doi: 10.1016/j. ajo.2016.06.041.

15. Ch'ng SW, Patton N, Ahmed M, Ivanova T, Baumann C, Charles S, et al. The Manchester Large Macular Hole Study: Is it time to reclassify large macular holes? Am J Ophthalmol. 2018;pii:S0002-9394(18)30408-2. doi: 10.1016/j. ajo.2018.07.027.

16. Yamashita T, Sakamoto T, Terasaki H, Iwasaki M, Ogushi $\mathrm{Y}$, Okamoto F, et al. Best surgical technique and outcomes for large macular holes: retrospective multicentre study in Japan. Acta Ophthalmol. 2018. doi: 10.1111/ aos.13795. [Epub ahead of print]

17. Gekka T, Watanabe A, Ohkuma Y, Arai K, Watanabe $\mathrm{T}$, Tsuzuki A. Pedicle Internal Limiting Membrane Transposition Flap Technique for Refractory Macular Hole. Ophthalmic Surg Lasers Imaging Retina. 2015;46(10):1045-1046. doi: 10.3928/2325816020151027-10.

18. Ozdek S, Baskaran P, Karabas L, Neves PP. A Modified Perfluoro-n-octane Assisted Autologous Internal Limiting Membrane Transplant for Failed Macular Hole Reintervention: A Case Series. Ophthalmic Surg Lasers Imaging Retina. 2017;48(5):416-420. doi: 10.3928/2325816020170428-08.

19. Chen SN, Yang CM. Double Internal Limiting Membrane Insertion for Macular Hole-Associated Retinal Detachment. J Ophthalmol. 2017;2017:3236516. doi: 10.1155/2017/3236516.

20. Baba R, Wakabayashi Y, Umazume K, Ishikawa T, Yagi H, Muramatsu D. Efficacy of the Inverted Internal Limiting Membrane Flap Technique with Vitrectomy for Retinal Detachment Associated with Myopic Macular Holes. Retina. 2017;37(3):466-471. doi: 10.1097/IAE.0000000000001211.

21. Rizzo S, Tartaro R, Barca F, Caporossi T, Bacherini D, Giansanti F. Internal Limiting Membrane Peeling Versus Inverted Flap Technique for Treatment of Full-Thickness Macular Holes: A Comparative Study in a Large Series of Patients. Retina. 2018;38(Suppl 1):S73-S78. doi: 10.1097/ IAE.0000000000001985.

\section{Author`s Contribution:}

HT primary surgeon \& conceived the design. AAK did data collection and manuscript writing. SJ did review of literature and proof reading. 